\begin{tabular}{|c|l|}
\hline Title & $\begin{array}{l}\text { In-Plane Orientation Control of 2,7-Diphenyl[1]benzothieno[3,2-b][1]benzothiophene Monolayer on Bismuth- } \\
\text { Terminated Si(111) Vicinal Surfaces with Wettability Optimization }\end{array}$ \\
\hline Author(s) & $\begin{array}{l}\text { Ohtomo, Manabu; T Suchida, Y uya; Muray a, Naoki; Y anase, Takashi; Sakai, Seiji; Y onezawa, Tetsu; Nagahama, Taro; } \\
\text { Hasegawa, Tetsuya; Shimada, Toshihiro }\end{array}$ \\
\hline Citation & $\begin{array}{l}\text { The Journal of Physical Chemistry C, 117(22), 11555-11561 } \\
\text { https://doi.org/10.1021/jp3117837 }\end{array}$ \\
\hline Issue Date & 2013-06-06 \\
\hline Doc URL & http://hdl.handle.net/2115/56245 \\
\hline Type & article \\
\hline File Information & jp3117837.pdf \\
\hline
\end{tabular}

Instructions for use 


\title{
In-Plane Orientation Control of 2,7- Diphenyl[1]benzothieno[3,2-b][1]benzothiophene Monolayer on Bismuth-Terminated Si(111) Vicinal Surfaces with Wettability Optimization
}

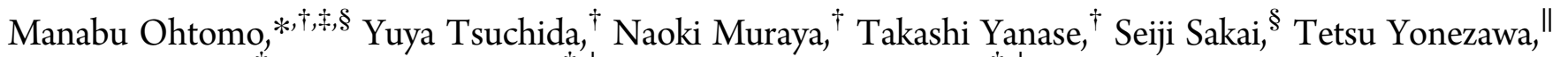 \\ Taro Nagahama, ${ }^{\dagger}$ Tetsuya Hasegawa, ${ }^{\ddagger}, \perp$ and Toshihiro Shimada $*, \dagger, \perp$ \\ ${ }^{\dagger}$ Division of Materials Chemistry, Faculty of Engineering, Hokkaido University, Kita 13 Nishi 8, Sapporo, Hokkaido 060-8628, Japan \\ ${ }^{\ddagger}$ Department of Chemistry, Graduate School of Science, The University of Tokyo, Hongo 7-3-1, Tokyo 113-0033, Japan \\ ${ }^{\S}$ Advanced Science Research Center, Japan Atomic Energy Agency, 2-4 Shirakata-Shirane, Tokai, Ibaraki 319-1195, Japan \\ "Division of Materials Science and Engineering, Faculty of Engineering, Hokkaido University, Kita 13 Nishi 8, Sapporo, Hokkaido \\ 060-8628, Japan \\ ${ }^{\perp}$ CREST-JST, Gobancho, Chiyoda-ku, Tokyo 102-0076, Japan
}

ABSTRACT: We report the in-plane orientation control of a high-mobility organic semiconductor: 2,7-diphenyl[1] benzothieno[3,2- $b][1]$ benzothiophene (DPh-BTBT). As previously reported for the monolayer pentacene, it was revealed that bunched steps on the vicinal $\mathrm{Si}(111)$ with a bismuth termination break the surface 3-fold symmetry and reduce the multiple orientation of the $\mathrm{DPh}-\mathrm{BTBT}$ grains into a quasi-single orientation. Interestingly, the critical step height necessary for the orientation control was higher than that of pentacene. We examined several mechanisms of orientation control and concluded that the facet nanostructure fabricated by step bunching works as an anisotropic template for the nucleation. We also show the wettability optimization of the bismuth-terminated silicon surface and show that the growth mode of $\mathrm{DPh}$ BTBT is dependent on the surface nanostructure of $\mathrm{Bi}-\mathrm{Si}$.
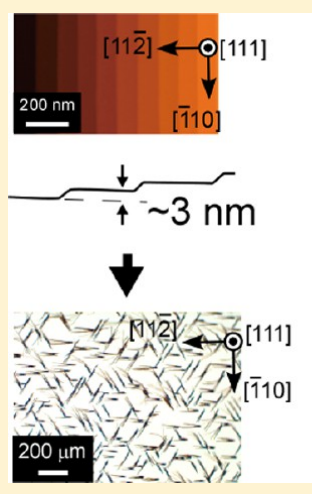
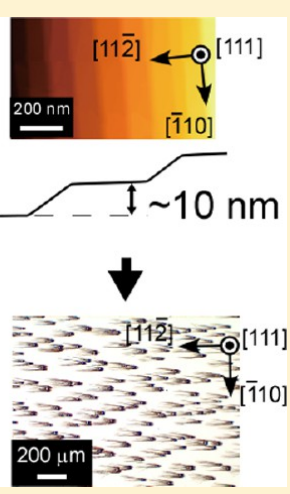

\section{INTRODUCTION}

As intensive efforts have been made to design novel highmobility organic semiconductor molecules, there are growing numbers of newly synthesized molecules with a carrier mobility higher than $1 \mathrm{~cm}^{2} /(\mathrm{V} \mathrm{s})$. The benzothieno-benzothiophene derivatives, for example, are attracting much attention for their high mobility, air-stability, and good crystallinity. ${ }^{1,2}$ In order to develop an efficient strategy to design high mobility molecules, their fundamental parameters for carrier transport, such as transfer integrals, effective mass, and carrier-phonon coupling constant, should be evaluated through photoemission spectroscopy.

We have previously reported the epitaxial growth of the quasi-single crystalline pentacene with a "thin-film" phase polymorph on Bi-terminated $\operatorname{Si}(111)$ vicinal surfaces with a $\sqrt{ } 3$ $\times \sqrt{3}$ reconstruction. ${ }^{3}$ Bismuth was previously found to be an ideal atom to terminate the surface dangling bonds of silicon, because the $\mathrm{Bi}-\mathrm{Si}(111)$ surface is inert enough to grow pentacene in a "standing" orientation. ${ }^{4}$ It is the same orientation as those in practical field effect transistor (FET) devices, in which the molecular long axis of pentacene is almost perpendicular to the substrate surface. This is in clear contrast with metallic surfaces, on which a strong interaction between metal orbitals and molecular $\pi$ orbitals make the molecules "lying". In our previous report, we have carried out the angleresolved photoemission spectroscopy (ARPES) studies of the "thin-film" phase monolayer pentacene on $\mathrm{Bi}-\mathrm{Si}(111)$ $(\sqrt{3} \times \sqrt{3})$ and determined some of the important parameters like the effective mass and transfer integrals. ${ }^{5}$

However, the detailed mechanism of the quasi-singleoriented growth was not thoroughly understood, even though some possible mechanisms were suggested. Because the $\mathrm{Bi}-$ $\operatorname{Si}(111)-(\sqrt{3} \times \sqrt{3})$ reconstructed surface has a 3 -fold symmetry and the pentacene crystal has a triclinic lattice, it is more likely to obtain domains with six in-plane orientations in the epitaxial films. Our essential finding was that step bunching in the vicinal substrates seems to play an important role.

In this article, we report that the in-plane orientation control of 2,7-diphenyl[1]benzothieno[3,2-b][1] benzothiophene (DPh-BTBT; Figure 1a) is indeed possible as well on bismuth terminated $\mathrm{Si}(111)$ vicinal surfaces with bunched steps. We will also demonstrate that the wettability of DPh-BTBT is notably dependent on the $\mathrm{Bi}-\mathrm{Si}$ nanostructure. By comparing the requirement for orientation control between pentacene and

Received: November 30, 2012

Revised: May 10, 2013

Published: May 13, 2013 
a
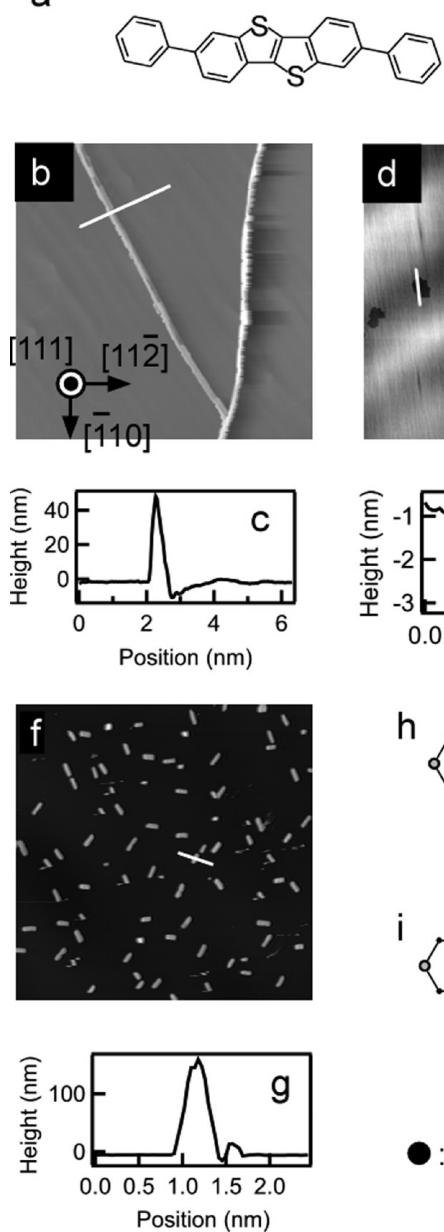

Figure 1. (a) Structural formula of 2,7-diphenyl[1] benzothieno[3,2$b][1]$ benzothiophene (DPh-BTBT). (b-g) AFM images and their cross section profiles of DPh-BTBT crystals grown on (b, c) $\beta$-phase (1 ML Bi), (d, e) $\alpha$-phase (1/3 ML Bi), and (f, g) mixed phase (0.66 ML). (h, i) Schematic diagram of (h) $\beta$-phase and (i) $\alpha$-phase Bi$\operatorname{Si}(111)-(\sqrt{3} \times \sqrt{3})$.

DPh-BTBT, we discuss the possible mechanism of orientation control on vicinal silicon surfaces with a heteroatom termination.

\section{EXPERIMENTAL METHODS}

The substrate was $\mathrm{Si}(111)$ with miscuts in various angles and directions. It was cleaned following the Shiraki method. ${ }^{6}$ The sample was mounted on a sample holder made of Mo, and introduced into a vacuum chamber equipped with a Knudsen cell, pyrometer, and reflection high energy electron diffraction with a microchannel plate (MCP-RHEED). ${ }^{7}$ The acceleration voltage and the typical sample current were $15 \mathrm{kV}$ and $100 \mathrm{pA}$, respectively. First, the sample was thoroughly degassed overnight at $450{ }^{\circ} \mathrm{C}$ and then flashed at $1260{ }^{\circ} \mathrm{C}$ for $20 \mathrm{~s}$. At $860{ }^{\circ} \mathrm{C}$, the $1 \times 1$ reflection pattern turned into the $7 \times 7$ reconstruction, which was confirmed by RHEED. At this point, the pyrometer was calibrated to $860{ }^{\circ} \mathrm{C}$. From 1260 to $960{ }^{\circ} \mathrm{C}$, the sample was rapidly cooled in $1 \mathrm{~min}$ in order to avoid silicon carbide formation. From 960 to $800{ }^{\circ} \mathrm{C}$, the sample was slowly cooled at the rate of $8{ }^{\circ} \mathrm{C} / \mathrm{min}$ in order to promote step bunching. ${ }^{8}$ All of these processes were performed under $<2 \times$ $10^{-9}$ Torr vacuum conditions. Monolayer equivalent bismuth was then deposited from the Knudsen cell onto the substrate at room temperature, and the $1 \times 1$ RHEED pattern was confirmed.

To turn $1 \times 1$ Bi into the $\sqrt{ } 3 \times \sqrt{ } 3$ reconstructed surface, the sample was annealed after the Bi deposition. The coverage of $\mathrm{Bi}$ was estimated using ex situ X-ray photoemission spectroscopy by comparing the intensity ratio of the $\mathrm{Bi} 4 \mathrm{f}_{7 / 2}$ and $\mathrm{Si} 2 \mathrm{p}$ peak intensities. DPh-BTBT molecules, used as purchased, were subsequently deposited from the Knudsen cell. The substrate temperature was kept at $80{ }^{\circ} \mathrm{C}$ during the growth, which was the optimum value in terms of wettability and crystallinity.

Parts of the films were examined by Grazing Incidence X-ray Diffraction (GIXD; Rigaku SmartLab $9 \mathrm{~kW}$ with $\mathrm{CuK} \alpha$ ) under ambient conditions to determine the precise geometry of the reciprocal lattice.

\section{RESULTS AND DISCUSSION}

We first discuss the growth mode of the DPh-BTBT monolayer on the bismuth terminated silicon surface. Figure 1 shows the AFM images of the DPh-BTBT crystals grown on $\mathrm{Bi}-\mathrm{Si}(111)$ with three surface nanostructures. It was reported that the post annealing temperature after the bismuth deposition is crucial for the nanostructure of the $\mathrm{Bi}-\mathrm{Si}$ termination..$^{9-12}$ When annealed at $350{ }^{\circ} \mathrm{C}$, the surface dangling bonds are terminated by $1 \mathrm{ML}$ bismuth and called the $\beta$-phase (so-called milk-stool model, Figure $1 \mathrm{~h}$ ). On the other hand, annealing at $597{ }^{\circ} \mathrm{C}$ leads to low coverage of $1 / 3 \mathrm{ML}$, which is called the $\alpha$-phase (Figure1i). The annealing temperature between 350 and 590 ${ }^{\circ} \mathrm{C}$ results in a mixture of $\alpha$ - and $\beta$-phases, which we call the mixed phase $(\sim 0.66 \mathrm{ML})$. As is clearly shown in Figure $1 \mathrm{~b}-\mathrm{g}$, the growth mode of the DPh-BTBT monolayer was dependent on the phases of the $\mathrm{Bi}-\mathrm{Si}$ surfaces. For the $\beta$ - and mixed phase, the monolayer equivalent DPh-BTBT was grown in the Volmer-Weber (VW) mode, that is, three-dimensional growth resulting in bulky needle crystals aligned in three directions, which reflect the surface 3 -fold symmetry of the $\mathrm{Bi}-\mathrm{Si}(111)$ surface. On the other hand, the $\alpha$-phase surface seems to provide a lower contact angle with the DPh-BTBT films. The DPh-BTBT film was grown in a layer-by-layer mode in the first monolayer, followed by an island growth in the second layer and above (Stranski-Klastanow (SK) mode). This is in contrast to the growth of pentacene on $\mathrm{Bi}-\mathrm{Si}(111)$; pentacene films were grown in the VW mode on the $\beta$-phase and in the SK mode on the $\alpha$ - and mixed phases.

The reason for the different wettability can be attributed to the surface energies of the $\alpha$-, $\beta$-, and mixed phase surfaces. In the droplet model of the film growth, which we now adopt as a crude approximation, the contact angle $\theta$ of the adsorbed "droplet" can be determined as follows:

$$
\cos \theta=\frac{\gamma_{\mathrm{s}}-\gamma_{\mathrm{i}}}{\gamma_{\mathrm{f}}}
$$

where $\gamma_{\mathrm{f}}, \gamma_{\mathrm{s}}$, and $\gamma_{\mathrm{i}}$ are the surface energy of the organic film, substrate surface, and interface energy, respectively.

To the best of our knowledge, there are no reports on the interface energy of $\mathrm{DPh}-\mathrm{BTBT} / \mathrm{Bi}-\mathrm{Si}(111)$. Still, we can estimate the order of magnitude of $\gamma_{\mathrm{i}}$ by taking the adsorption energy of analogous systems into consideration. It was previously reported that the adsorption energy of pentacene on graphite was $1.14 \mathrm{eV} .{ }^{13}$ By taking the unit cell area of 110.1 $\AA^{2}$ into account, this gives an interface energy of $0.17 \mathrm{~J} / \mathrm{m}^{2}$. Because the interaction between $\mathrm{DPh}-\mathrm{BTBT}$ and $\mathrm{Bi}-\mathrm{Si}(111)$ is 
a van der Waals interaction, it is reasonable to assume that the interface energy of DPh-BTBT/Bi-Si(111) is on the same order of magnitude as, or even lower than, that of the pentacene/HOPG interface.

The surface energy of the $\alpha$ - and $\beta$-phase $\mathrm{Bi}-\mathrm{Si}(111)$, on the other hand, was calculated by Kunc and co-workers using the density functional theory. ${ }^{14}$ The bismuth adsorption energy was evaluated by the energy gain of the system with respect to the total energy of the ideal (unreconstructed) $\mathrm{Si}(111)-(1 \times 1)$ surface and isolated $\mathrm{Bi}$ atom. Interestingly, it was revealed that the $\alpha$-phase $\mathrm{Bi}-\mathrm{Si}$ is more unstable than the $\beta$-phase by $4.6 \mathrm{~J} /$ $\mathrm{m}^{2}$, while $\mathrm{Si}(111)-(7 \times 7)$ is even more unstable. It should be noted that the energy gain of $4.6 \mathrm{~J} / \mathrm{m}^{2}$ is 1 order of magnitude greater than the interface energy of the pentacene/graphite. Therefore, it is reasonable to assume that the increase in the surface energy of the $\alpha$-phase lowers the contact angle between the DPh-BTBT and $\mathrm{Bi}-\mathrm{Si}$ heterointerface.

It is relatively easy to qualitatively understand the instability of the $\alpha$-phase. The $\alpha$-phase surface is a considerably strained surface as previously reported. ${ }^{10}$ The largest displacement of the $\mathrm{Si}$ atoms in the $\alpha$-phase reconstruction is as high as $0.20 \AA$, while that in the $\beta$-phase milk stool model is $0.11 \AA$. The instability of the $\alpha$-phase is also reinforced by the fact that it can only be fabricated in a very narrow temperature range. The Bi atoms were easily desorbed by annealing at a temperature higher than $600{ }^{\circ} \mathrm{C}$, which indicated the instability of the $\alpha$ phase.

In the case of pentacene, on the other hand, the SK mode growth was also observed on the mixed phase surfaces. We have examined the growth of pentacene and DPh-BTBT on three types of $\mathrm{Bi}-\mathrm{Si}(111)$ substrates $(\alpha-, \beta$-, and mixture with different ratios) at various substrate temperatures and growth rates, and typical examples are described in this paper. The results indicated that the $\mathrm{DPh}-\mathrm{BTBT}(001)$ surface is more unstable than the pentacene (001) surface, that is, DPh-BTBT molecules are more likely to segregate than the pentacene molecules, at elevated temperatures and on the $\alpha$ - and $\beta$-mixed surfaces.

Before discussing the in-plane orientation of DPh-BTBT on the bismuth terminated $\mathrm{Si}(111)$, we will briefly summarize our previous reports on the growth of the quasi-single crystalline pentacene monolayer on vicinal $\operatorname{Si}(111)$ with $\alpha$-phase bismuth termination. ${ }^{3}$ Because the $\mathrm{Bi}-\mathrm{Si}(111)-(\sqrt{3} \times \sqrt{3})$ surface has a 3 -fold symmetry, the triclinic $\left(\gamma=89^{\circ}\right)$ pentacene monolayer has six in-plane orientations, in which each two of them are twin crystals. The fundamental idea is to break the surface symmetry of $\mathrm{Bi}-\mathrm{Si}(111)$. We employed the vicinally cut $\mathrm{Si}(111)$ surface inclined toward the $[11 \overline{2}]$ direction and found out that step bunching is important to reduce the orientation of the pentacene. The $3 \mathrm{~nm}$ steps formed on the $2^{\circ}$ off surface (Figure 2a) were required to reduce the in-plane orientation from 6 to 2, which are twin crystals. The role of the step bunching was not thoroughly understood, while the surface energy anisotropy of the pentacene model was considered unlikely, because the most unfavorable facet of pentacene crystal was parallel to the step edges.

$\mathrm{DPh}-\mathrm{BTBT}$ was also grown in the quasi-three orientations on the flat $\mathrm{Bi}-\mathrm{Si}(111)-(\sqrt{3} \times \sqrt{3})$. It was easy to recognize for the needle crystals on the $\beta$-phase $\mathrm{Bi}-\operatorname{Si}(111)-(\sqrt{3} \times \sqrt{3})$ (Figure 2d). For the $\alpha$-phase surface, on the other hand, there were no patterns or streaks confirmed with one monolayer thickness by MCP-RHEED. Another powerful method to determine the in-plane orientation of the monolayer grains was
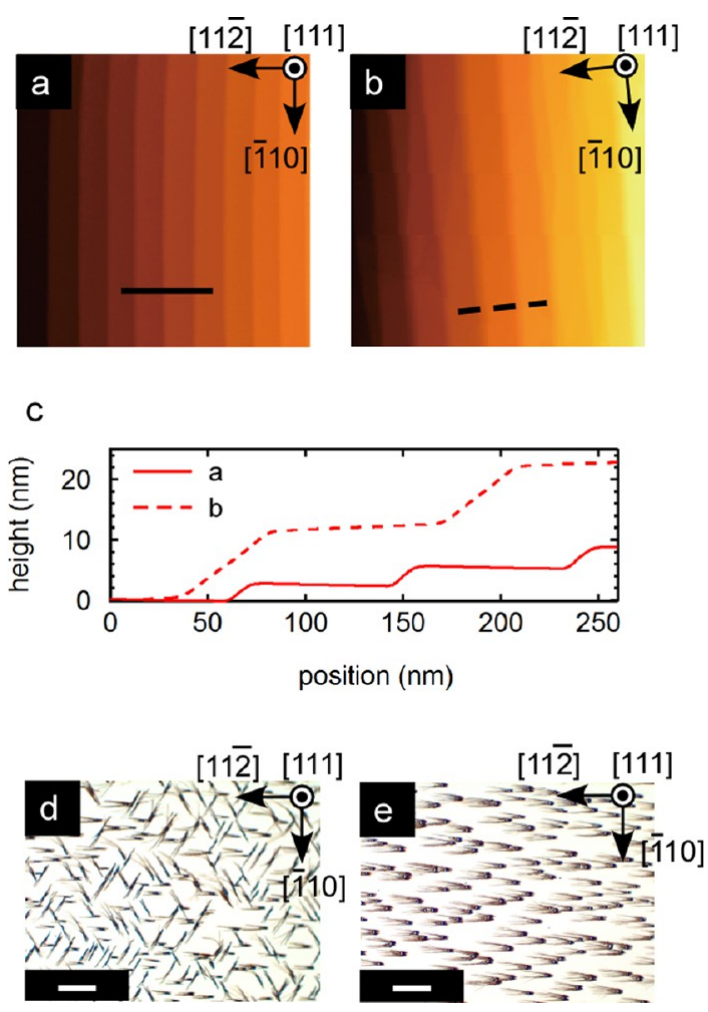

Figure 2. $(a-c)$ AFM images and their cross section profiles of periodic bunched steps on (a) $2^{\circ}$ off and (b) $4^{\circ}$ off vicinal $\mathrm{Si}(111)$ surfaces inclined toward the $[11 \overline{2}]$ direction. Steps with 3 and $10 \mathrm{~nm}$ heights can be fabricated from $2^{\circ}$ off and $4^{\circ}$ off vicinal surface, respectively, through gradual cooling of the sample after $1260{ }^{\circ} \mathrm{C}$ flash heating. (d, e) Optical microscope image of DPh-BTBT needle crystals grown on $\beta$-phase $\mathrm{Bi}-\mathrm{Si}(111)-(\sqrt{3} \times \sqrt{3})$ with (d) flat surface and (e) $10 \mathrm{~nm}$ steps $($ scale bar $=200 \mu \mathrm{m})$. As shown in $(\mathrm{a}-\mathrm{c})$, $[11 \overline{2}]$ is the step descending direction.

recently developed by Frisbie and his co-workers. ${ }^{15,16}$ It is called transverse shear microscopy (TSM), analogous to friction force microscopy (FFM). The difference between TSM and FFM is the scanning direction. While friction parallel to the scanning direction is detected by conventional FFM, TSM utilizes the small transverse torsion of the cantilever which is perpendicular to the scanning direction. The TSM signal is high in the soft and anisotropic crystals like organic molecular crystals. It was reported that the TSM signal in the [110] direction is the highest in the herringbone packing structure, which enables us to determine the orientation of each grain in the polycrystalline pentacene films. As DPh-BTBT also has a herringbone packing, we have carried out the TSM study of the DPh-BTBT grains on flat $\alpha$-phase $\mathrm{Bi}-\mathrm{Si}(111)-(\sqrt{3} \times$ $\sqrt{ } 3)$. Figure $3 a, b$ shows the AFM and TSM images of the DPhBTBT monolayer on a flat substrate. It was confirmed that DPh-BTBT islands consisted of $\mu \mathrm{m}$ sized grains that are clearly shown in marked contrast difference in Figure $3 \mathrm{~b}$. There are three regions with discrete TSM signals: (i) $9 \pm 3 \mathrm{mV}$, (ii) -5 $\pm 2 \mathrm{mV}$, and (iii) $4 \pm 3 \mathrm{mV}$ with respect to the substrate surface. This result indicates that the DPh-BTBT islands consisted of grains with at least three distinct orientations. However, the DPh-BTBT crystals grown on $3 \mathrm{~nm}$ steps, though, did not show any significant change in the TSM mapping. We found that the critical step height necessary to reduce the number of TSM plateaus from three to one was 10 $\mathrm{nm}$, as indicated in Figure 3c,d, which was fabricated from $4^{\circ}$ 

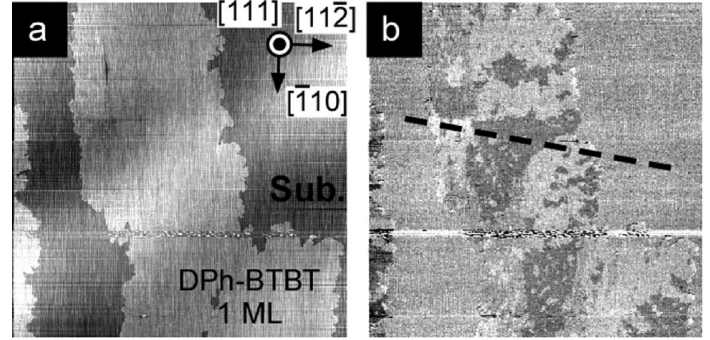

C
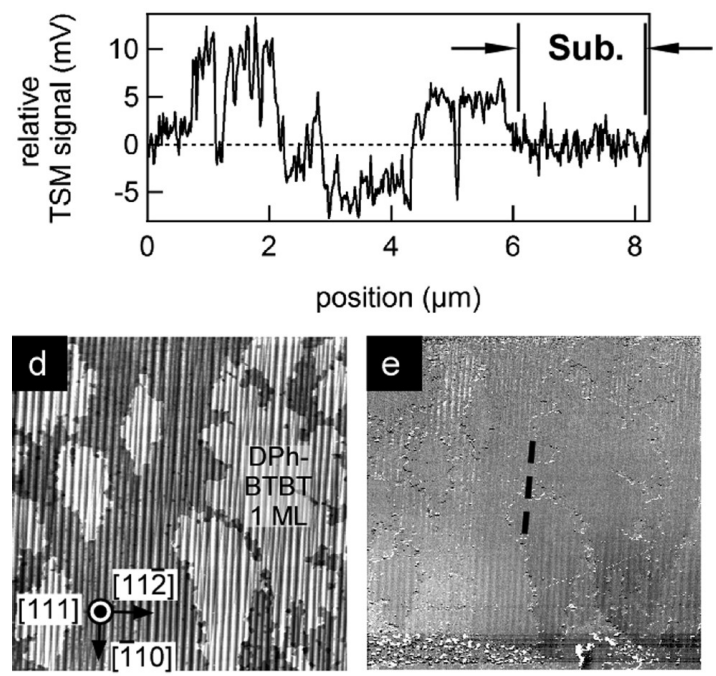

f

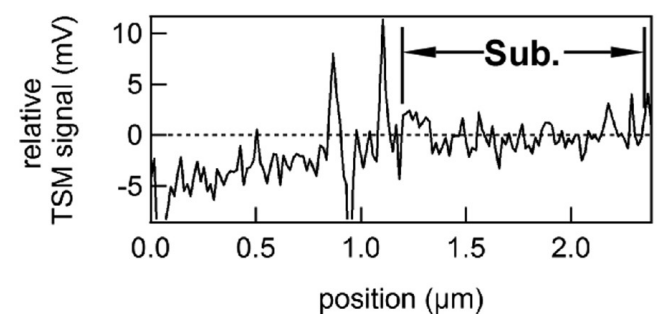

Figure 3. (a, b) AFM and TSM images of DPh-BTBT film on flat $1 / 3$ ML Bi-Si(111)- $(\sqrt{3} \times \sqrt{3})$, respectively. The monolayer island of DPh-BTBT in (a) was revealed to consist of several $\mu$ m-size grains with three discrete TSM signals as shown in (b). The cross section along a dashed line in (b) is plotted in (c). (d, e) AFM and TSM image in case of $4^{\circ}$ off-vicinal surface with 10 -nm steps. In the TSM image (e), there were no contrasts within or between the grains. The cross section shown in (f) indicates that the selected grains have TSM signals of $-5 \mathrm{mV}$ with respect to the substrate. All TSM signals were replotted with respect to the bare substrate surface. Scanning area is 8 $\times 8 \mu \mathrm{m}^{2}$ in all the images.

off-substrate inclined toward the $[11 \overline{2}]$ direction (Figure $2 b$ ). The TSM signals from the selected grains on the $10 \mathrm{~nm}$ steps were around $-5 \mathrm{mV}$ with respect to the bare substrate surfaces. To estimate the detailed orientation of the selected grains, we investigated the scanning orientation dependence. As we expected from the analogy to the pentacene orientation selection, we confirmed large TSM signals when the scanning direction was $40^{\circ}$ from the step edges (Figure 4), which implies that the $[100]$ or $[010]$ direction of DPh-BTBT lattice was aligned with the edges.

A more quantitative analysis of the aligned crystal structure was performed using MCP-RHEED and GIXD (Glazing Incidence $\mathrm{X}$-ray Diffraction) with the $\mathrm{X}$-ray incidence almost

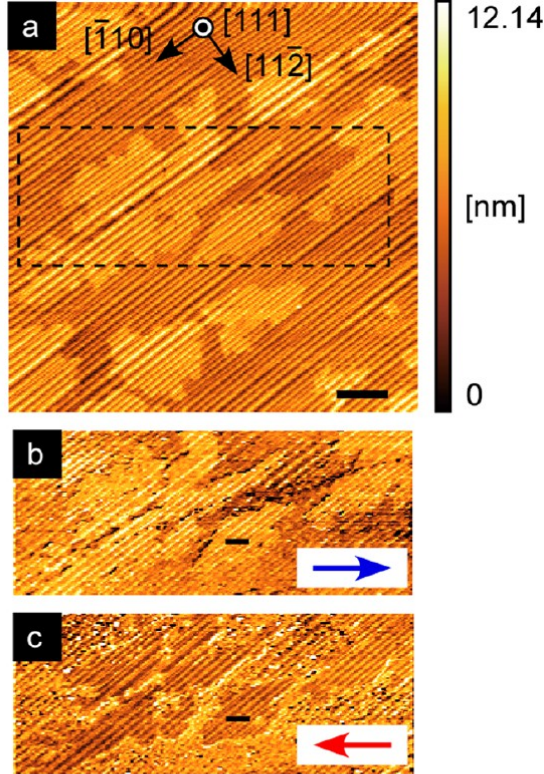

d

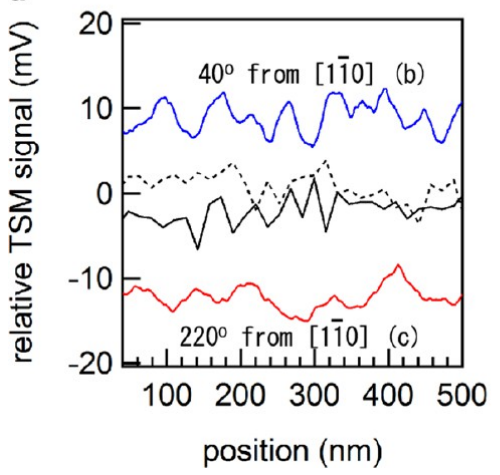

Figure 4. Scanning angle dependence of TSM signals of DPh-BTBT grains on $10 \mathrm{~nm}$ bunched steps. (a) Topography image (scale bar 1 $\mu \mathrm{m}$ ), (b) TSM image with scanning direction $40^{\circ}$, and (c) $220^{\circ}$ from $\mathrm{Si}[1 \overline{1} 0]$. The cross section plot of TSM signals with respect to the substrate is shown in (d). Black solid and dashed lines in (d) is the TSM profile along the $\mathrm{Si}[1 \overline{1} 0]$ and $\mathrm{Si}[1 \overline{1} 0]$, respectively. TSM signal along $\mathrm{Si}[1 \overline{1} 0]$ was around $0 \mathrm{mV}$ on average both in the forward and backward scans. Scanning at $40^{\circ}$ from $\mathrm{Si}[1 \overline{1} 10]$ direction showed a marked contrast between forward $\left(40^{\circ}\right)$ and backward $\left(220^{\circ}\right)$ scans. By assuming that the $\langle 110\rangle$ direction of the DPh-BTBT lattice has the highest TSM signal, we can estimate that the $\langle 100\rangle$ or $\langle 010\rangle$ direction of the DPh-BTBT lattice is parallel to the $\mathrm{Si}[1 \overline{1} 0]$ direction.

parallel to the surface. Figure 5a shows the MCP-RHEED pattern and Figure $5 b$ shows the GIXD pattern. In the MCPRHEED analysis, we obtained no apparent streaks or spots from DPh-BTBT on flat substrates. On the $4^{\circ}$ off-vicinal substrates with $10 \mathrm{~nm}$ height steps, on the other hand, spots derived from DPh-BTBT were observed as shown in Figure 5a when the incidence angle of the electron beam was perpendicular to the step edges (parallel to $\mathrm{Si}[11 \overline{2}]$ ). This result is consistent with the TSM analysis, and the observed spot can be assigned as the (02) diffraction of DPh-BTBT. The GIXD pattern revealed, however, a rather broad peak of the (20) diffraction of DPh-BTBT. This result implies a relatively large lattice mismatch between DPh-BTBT and $\mathrm{Bi}-\mathrm{Si}(111)$ $(\sqrt{3} \times \sqrt{3})$, resulting in an in-plane twisting up to $7^{\circ}$, corresponding to the broadening along the Phi direction in Figure $5 b$. 

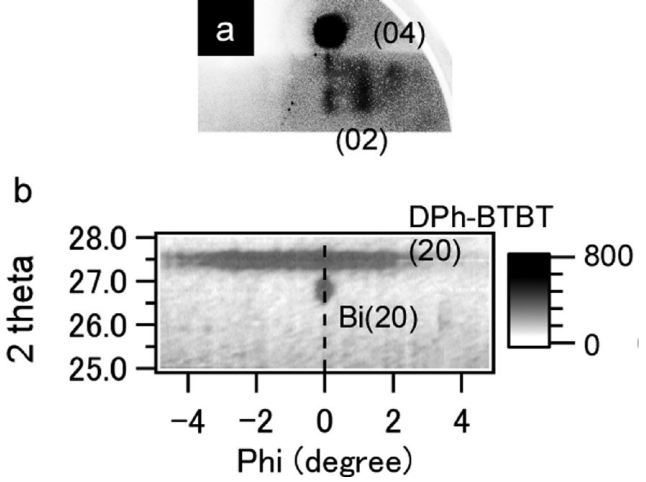

Figure 5. (a) MCP-RHEED and (b) GIXD patterns of monolayer DPh-BTBT films on $4^{\circ}$ off $1 / 3 \mathrm{ML} \mathrm{Bi}-\mathrm{Si}(111)-(\sqrt{3} \times \sqrt{3})$ with 10 $\mathrm{nm}$ steps. In the MCP-RHEED pattern, two spots assigned as the (02) and (04) diffractions were observed when the electron beam was parallel to $\mathrm{Si}[11 \overline{2}]$. In the GIXD reciprocal mapping, a narrow spot assigned to the $\mathrm{Bi}(20)$ diffraction and wide (20) diffraction of $\mathrm{DPh}$ BTBT were observed. The lattice constant derived from the (20) diffraction was $a=0.65 \mathrm{~nm}$. The phi in (b) was defined as the angle between the scattering vector of the Bragg reflection and $\mathrm{Si}[11 \overline{2}]$.

We propose three hypotheses for the orientation control mechanism on bunched steps. The first one is the kinetic selection of one orientation in which the speed of the crystal growth in the step descending or ascending direction prevail over those of the other directions. The second one is the mechanism similar to graphoepitaxy, which is a topographical effect of nanometer-size pitches or grooves on substrates. The last one is the "facet nucleation model". In this model, we assume that facets, which are formed in the bunched steps region, have an anisotropic surface structure and work as a template for crystal growth.

The kinetics model is based on the anisotropic growth rate of the crystals on inclined surfaces, that is, the speed of the crystal growth in a certain direction prevails over those of the others, resulting mostly in monodomain films. It was reported that atomic steps on vicinally cut substrates can induce an anisotropic growth rate of pentacene grains. ${ }^{17}$ Even though each step has only one atomic height, the growth rate of the pentacene monolayer was faster in the step-descending direction than in the step-ascending direction, resulting in dendritic branches in the step-descending direction and a compact shape in the opposite one. The possible mechanism of this anisotropic growth is a kinetic process. We have previously reported from a pulsed molecular beam scattering experiment on an inclined $\mathrm{H}-\mathrm{Si}(111)$ that surface residence time (SRT) was dependent on the direction of the incoming molecules: parallel to steps, ascending or descending. ${ }^{18,19}$ This result suggests that adsorption and desorption rates are not the same in the ascending and descending directions. Figure 6 depicts the needle crystals grown on $6 \mathrm{~nm}$ steps of $\beta-\mathrm{Bi}-\mathrm{Si}(111)-(\sqrt{3} \times$ $\sqrt{3})$. The crystals grown in the step ascending/descending $\left(0^{\circ}\right)$ direction perpendicular to the step edges were smaller than those in the other $\left( \pm 60^{\circ}\right)$ directions, which indicates the effect of the kinetic process. That is, the growth speed in the $0^{\circ}$ direction is slower than in the $\pm 60^{\circ}$ directions. When the step height was $10 \mathrm{~nm}$, it is expected that the diffusion across these higher steps will be suppressed. A simple consideration will lead to the more suppression of the needle crystals in the $0^{\circ}$ direction than those in the $\pm 60^{\circ}$ directions because of the higher necessity of diffusion across the steps. However, this
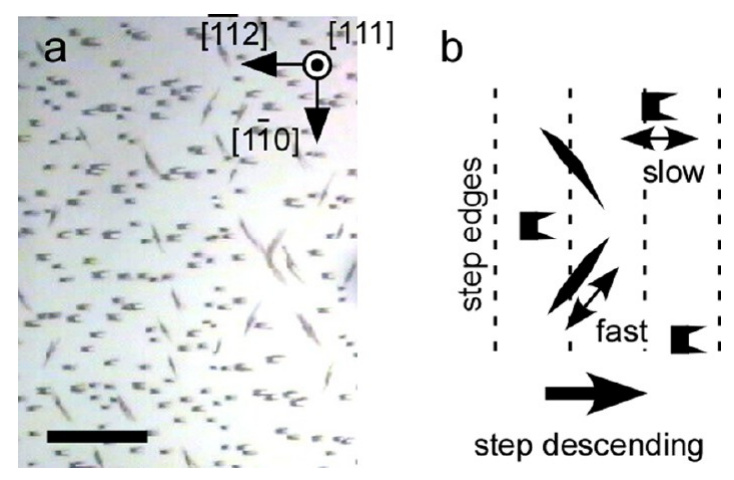

Figure 6. (a) Optical microscope image of DPh-BTBT needle crystals on $4^{\circ}$ off $\mathrm{Bi}-\mathrm{Si}(111)$ with $6 \mathrm{~nm}$ steps. The surface termination was confirmed to be the $\beta$-phase. Scale bar is $200 \mu \mathrm{m}$. (b) Schematic diagram of compact-shape crystals growing in step descending direction and long needle crystals in $\pm 60^{\circ}$ from $\mathrm{Si}[11 \overline{2}]$.

expectation is contrary to the actual result shown in Figure 2e. Hence, other factors must be considered. The orientation of flat monolayer domains on stepped surfaces (Figure 3e) also cannot be explained by simple kinetic effects because asymmetric contours as in ref 17 were not observed.

The second hypothesis, the graphoepitaxy model, is analogous to the previous reports of graphoepitaxy. ${ }^{20}$ To discuss this model, we present two results on the DPh-BTBT growth. First, it was revealed that the vicinal $\mathrm{Si}(111)$ inclined in $[11 \overline{0}]$ direction does not affect the orientation of the DPhBTBT monolayer. Even when the steps were larger than $10 \mathrm{~nm}$, the crystals were grown in three directions independent of the steps. Second, the antimony-terminated surface did not affect the growth of the crystals as well, regardless of the step height. The antimony terminated $\mathrm{Si}(111)$ was prepared following a previously reported procedure, ${ }^{21}$ which was reconstructed with the same $\sqrt{3} \times \sqrt{3}$ periodicity. Even though the steps were preserved after the antimony termination, steps did not affect the crystal growth at all. These two results indicate that the existence of only the geometrical guiding steps does not align the orientation of the DPh-BTBT lattice. That is, the atomic structure of the steps plays an important role.

The remaining hypothesis is the "facet nucleation model" illustrated in Figure 7. We consider that it can provide a reasonable explanation without any inconsistency. It is based on the accidental lattice matching of the growing molecules with that of the facets. For the bare $\operatorname{Si}(111)-(7 \times 7)$, high resolution scanning tunneling microscopy (STM) studies revealed that the bunched steps form $\mathrm{Si}(331)$ facets in the $10^{\circ}$ off-Si(111) surface, ${ }^{22-24}$ while no obvious periodicity was observed in the $2^{\circ}$ off-surface. $^{25} \mathrm{Si}(331)$ is the only stable surface existing between $\mathrm{Si}(111)$ and $\mathrm{Si}(110)$, as a consequence of the stable 12 $\times 1$ reconstruction. This hypothesis is based on the fact that the surface lattice constant of $\mathrm{Si}(331)-(1 \times 1)$ is $0.384 \times 0.860$ $\mathrm{nm}^{2}$, which matches very well with the lattice constant of the thin-film phase pentacene $(b=0.759 \mathrm{~nm})$ with a mismatch of $1 \%$. Therefore, it is reasonable to assume that the $\mathrm{Si}(331)$ or analogous nanostructure in the facets is working as an anisotropic template for the crystal growth. For DPh-BTBT, although the single crystal structure has not yet been solved yet, our MCP-RHEED analysis of the (11) diffraction revealed $b=$ $0.75 \mathrm{~nm}$, which gives a $2.4 \%$ mismatch with $\mathrm{Si}(331)-(1 \times 1)$.

The mechanism of the orientation with this model is as follows. The molecular crystals (pentacene or DPh-BTBT) 


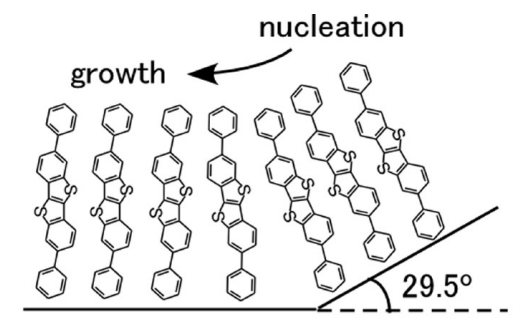

Terraces

Facets

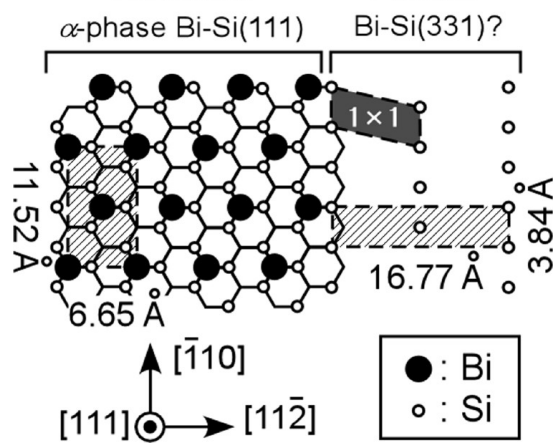

Figure 7. Schematic diagram explaining the "facet nucleation model". The facets assuming a $\mathrm{Si}(331)$ structure are superimposed on the terraces.

nucleates only on the facets because of the excellent lattice matching with the facet lattice. Nevertheless, if the critical nuclei size is greater than the facet size, nucleation will not occur. Because the height of the steps is proportional to the facet size, the probability of the nucleation is governed by the step heights. It is understood that the alignment of pentacene requires a smaller step height than DPh-BTBT because the lattice matching of pentacene is much better.

The effects of bismuth adsorption onto the facets of the vicinal $\mathrm{Si}(111)$ have not yet been investigated. However, we reinforce our hypothesis by presenting two additional experimental results as follows, which will be described in detail in a forthcoming paper. First, $\mathrm{Bi}-\mathrm{Si}(331)$ have a stable surface with $5 \times 1$ reconstruction. Second, $\mathrm{Sb}-\mathrm{Si}(331)$ do not have a stable surface reconstruction. The RHEED pattern of $\mathrm{Sb}-\mathrm{Si}(331)$ was that of the amorphous halo pattern until the desorption. This is probably the reason why the Sb-terminated vicinal surface did not affect the in-plane orientation; it did not have a nucleation template on the facets. Whereas a full understanding of the mechanism requires high-resolution STM studies of the bismuth terminated $\mathrm{Si}(111)$ vicinal surfaces, the most reasonable hypothesis presented so far seems to be the "facet nucleation model".

\section{CONCLUSION}

In this article, we have demonstrated that the growth mode and crystal orientation of DPh-BTBT is controlled through manipulating the nanostructure of the bismuth terminated $\mathrm{Si}(111)$ surface. The growth mode was significantly dependent on the coverage of the bismuth atoms; Volmer-Weber mode on 1 ML bismuth ( $\beta$-phase) and Stranski-Klastanow mode on $1 / 3$ ML ( $\alpha$-phase). The reason for this difference can be attributed to the high surface energy of the $\alpha$-phase surface, which is derived from the strained structure of the $\mathrm{Bi}-\mathrm{Si}$ termination. It was also revealed that bunched steps as high as $10 \mathrm{~nm}$ on the $4^{\circ}$ off-vicinal surfaces inclined toward the $[11 \overline{2}]$ direction are necessary to make quasi-single crystalline $\mathrm{DPh}$ -
BTBT films on $\mathrm{Bi}-\mathrm{Si}(111)-(\sqrt{3} \times \sqrt{3})$, while pentacene needed $3 \mathrm{~nm}$ steps as previously reported. The mechanism for orientation control and critical step height difference was successfully explained by the "facet nucleation model", in which the facet structure in bunched steps, possibly analogous to $\mathrm{Si}(331)$, plays an important role. This result enables us to fabricate films with a well-defined crystal orientation on conductive substrates and accelerate spectroscopic studies of newly developed organic semiconductors.

\section{AUTHOR INFORMATION}

\section{Corresponding Author}

*Tel.: +81-29-284-3802 (M.O.); +81-11-706-6576 (T.S.). Email: ohtomo.manabu@jaea.go.jp (M.O.); shimadat@eng. hokudai.ac.jp (T.S.).

\section{Notes}

The authors declare no competing financial interest.

\section{ACKNOWLEDGMENTS}

We thank Prof. Kazuo Takimiya (Hiroshima Univ.) for his fruitful discussion. This work was supported by the Global Centers of Excellence (GCOE) Program "Chemistry Innovation through Cooperation of Science and Engineering" and Grant-in-Aid for JSPS Fellows from the Japan Society for the Promotion of Science (JSPS).

\section{REFERENCES}

(1) Yamamoto, T.; Takimiya, K. Facile Synthesis of Highly PiExtended Heteroarenes, Dinaphtho[2,3-b: 2 ',3 '-f]chalcogenopheno[3,2-b]chalcogenophenes, and Their Application to Field-Effect Transistors. J. Am. Chem. Soc. 2007, 129, 2224-2225.

(2) Takimiya, K.; Ebata, H.; Sakamoto, K.; Izawa, T.; Otsubo, T.; Kunugi, Y. 2,7-Diphenyl[1] benzothieno[3,2-b] benzothiophene, a New Organic Semiconductor for Air-Stable Organic Field-Effect Transistors with Mobilities up to $2.0 \mathrm{~cm}^{2} \mathrm{~V}^{-1} \mathrm{~s}^{-1}$. J. Am. Chem. Soc. 2006, 128 , 12604-12605.

(3) Shimada, T.; Ohtomo, M.; Suzuki, T.; Hasegawa, T.; Ueno, K.; Ikeda, S.; Saiki, K.; Sasaki, M.; Inaba, K. Step-Bunched Bi-Terminated $\mathrm{Si}(111)$ Surfaces as a Nanoscale Orientation Template for Quasisingle Crystalline Epitaxial Growth of Thin Film Phase Pentacene. Appl. Phys. Lett. 2008, 93, 223303.

(4) Sadowski, J. T.; Nagao, T.; Yaginuma, S.; Fujikawa, Y.; AlMahboob, A.; Nakajima, K.; Sakurai, T.; Thayer, G. E.; Tromp, R. M. Thin Bismuth Film as a Template for Pentacene Growth. Appl. Phys. Lett. 2005, 86, 073109 .

(5) Ohtomo, M.; Suzuki, T.; Shimada, T.; Hasegawa, T. Band Dispersion of Quasi-Single Crystal Thin Film Phase Pentacene Monolayer Studied by Angle-Resolved Photoelectron Spectroscopy. Appl. Phys. Lett. 2009, 95, 123308.

(6) Ishizaka, A.; Shiraki, Y. Low-Temperature Surface Cleaning of Silicon and its Application to Silicon MBE. J. Electrochem. Soc. 1986, $133,666-671$.

(7) Saiki, K.; Kono, T.; Ueno, K.; Koma, A. Highly Sensitive Reflection High-Energy Electron Diffraction Measurement by Use of Micro-Channel Imaging Plate. Rev. Sci. Instrum. 2000, 71, 3478-3479.

(8) Lin, J. L.; Petrovykh, D. Y.; Viernow, J.; Men, F. K.; Seo, D. J.; Himpsel, F. J. Formation of Regular Step Arrays on $\mathrm{Si}(111) 7 \times 7$. J. Appl. Phys. 1998, 84, 255-260.

(9) Takahashi, T.; Izumi, K.; Ishikawa, T.; Kikuta, S. Evidence for a Trimer in the $\sqrt{3} \times \sqrt{3}$-Bi Structure on the $\mathrm{Si}(111)$ Surface by XRay-Diffraction under the Nearly Normal Incidence Condition. Surf. Sci. 1987, 183, L302-L312.

(10) Kuzumaki, T.; Shirasawa, T.; Mizuno, S.; Ueno, N.; Tochihara, H.; Sakamoto, K. Re-Investigation of the Bi-Induced $\mathrm{Si}(111)-(\sqrt{3} \times$ $\sqrt{3}$ ) Surfaces by Low-Energy Electron Diffraction. Surf. Sci. 2010, 604, 1044-1048. 
(11) Shioda, R.; Kawazu, A.; Baski, A. A.; Quate, C. F.; Nogami, J. Bi on $\operatorname{Si}(111)$ - Two Phases of the $\sqrt{3} \times \sqrt{3}$ Surface Reconstruction. Phys. Rev. B: Condens. Matter Mater. Phys. 1993, 48, 4895-4898.

(12) Wan, K. J.; Guo, T.; Ford, W. K.; Hermanson, J. C. Low-Energy Electron-Diffraction Studies of $\mathrm{Si}(111)-\sqrt{3} \times \sqrt{3}-\mathrm{R} 30^{\circ}$-Bi System Observation and Structural Determination of Two Phases. Surf. Sci. 1992, 261, 69-87.

(13) Götzen, J.; Käfer, D.; Wöll, C.; Witte, G. Growth and Structure of Pentacene Films on Graphite: Weak Adhesion as a Key for Epitaxial Film Growth. Phys. Rev. B: Condens. Matter Mater. Phys. 2010, 81, 085440 .

(14) Cheng, C.; Kunc, K. Structure and Stability of Bi Layers on $\mathrm{Si}(111)$ and $\mathrm{Ge}(111)$ Surfaces. Phys. Rev. B: Condens. Matter Mater. Phys. 1997, 56, 10283-10288.

(15) Kalihari, V.; Haugstad, G.; Frisbie, C. D. Distinguishing Elastic Shear Deformation from Friction on the Surfaces of Molecular Crystals. Phys. Rev. Lett. 2010, 104, 086102.

(16) Kalihari, V.; Tadmor, E. B.; Haugstad, G.; Frisbie, C. D. Grain Orientation Mapping of Polycrystalline Organic Semiconductor Films by Transverse Shear Microscopy. Adv. Mater. 2008, 20, 4033-4039.

(17) Nishikata, S.; Sazaki, G.; Takeuchi, T.; Usami, N.; Suto, S.; Nakajima, K. Step-Induced Anisotropic Growth of Pentacene Thin Film Crystals on a Hydrogen-Terminated $\mathrm{Si}(111)$ Surface. Cryst. Growth Des. 2007, 7, 439-444.

(18) Shimada, T.; Ichikawa, H.; Saiki, K. Pulsed Molecular Beam Scattering of a Planar-Shaped Organic Molecule on Regularly Stepped Surfaces of Hydrogen-Terminated Si(111). Appl. Phys. Lett. 2006, 89, 141912.

(19) Shimada, T.; Ichikawa, H.; Koma, A.; Saiki, K. Interaction between Surface Migrating Pentacene Molecules and Chemically Modified Surfaces of Silicon Oxides Studied by Pulsed Molecular Beam Scattering. Surf. Sci. 2006, 600, L236-L239.

(20) Ikeda, S.; Saiki, K.; Wada, Y.; Inaba, K.; Ito, Y.; Kikuchi, H.; Terashima, K.; Shimada, T. Graphoepitaxy of Sexithiophene and Orientation Control by Surface Treatment. J. Appl. Phys. 2008, 103, 084313.

(21) Abukawa, T.; Park, C. Y.; Kono, S. Photoelectron Diffraction Study of the Atomic Geometry of the $\operatorname{Si}(111) \sqrt{3} \times \sqrt{3}-$ Sb Surface. Surf. Sci. 1988, 201, L513-L518.

(22) Hibino, H.; Ogino, T. Transient Step Bunching on a Vicinal Si(111) Surface. Phys. Rev. Lett. 1994, 72, 657.

(23) Suzuki, M.; Homma, Y.; Hibino, H.; Fukuda, T.; Sato, T.; Iwatsuki, M.; Miki, K.; Tokumoto, H. Real-Time Scanning-TunnelingMicroscopy of Phase-Transition and Faceting on a Vicinal Si(111) Surface. J. Vac. Sci. Technol., A 1993, 11, 1640-1643.

(24) Homma, Y.; McClelland, R. J.; Hibino, H. DC-ResistiveHeating-Induced Step Bunching on Vicinal Si(111). Jpn. J. Appl. Phys., Part 2 1990, 29, L2254-L2256.

(25) Men, F. K.; Liu, F.; Wang, P. J.; Chen, C. H.; Cheng, D. L.; Lin, J. L.; Himpsel, F. J. Self-Organized Nanoscale Pattern Formation on Vicinal $\mathrm{Si}(111)$ Surfaces via a Two-Stage Faceting Transition. Phys. Rev. Lett. 2002, 88, 096105. 